\title{
The Functional Behaviors of Cosurfactant in Design of Self-nanoemulsifying Drug Delivery Systems
}

\author{
Su-Geun Yang ${ }^{1,2 \dagger}$ and Hee-Jong Shin $^{3}$ \\ ${ }^{1}$ Utah-Inha DDS and Advanced Therapeutics, Annex B-403, Meet-You-All Tower, Songdo Technopark, 7-50 \\ Songdo-dong, Yeonsu-gu, Incheon 406-840, South Korea \\ ${ }^{2}$ Clinical Research Center, School of Medicine, Inha University, 2F A-dong, Jeongseok Bldg., \\ Sinheung-dong 3-ga, Jung-gu, Incheon 400-712, South Korea \\ ${ }^{3}$ CKD Research Institute, Chong Kun Dang Pharm. Co., 15-20 Osackdang-Ri, Seonggeo-Up, \\ Cheonan-Shi, Chungcheongnam-Do 330-830, South Korea
}

(Received July 21, 2010 • Revised August 12, $2010 \cdot$ Accepted August 13, 2010)

\begin{abstract}
Nanoemulsions have been widely investigated for many years because of their attractive and unique characteristics. Nanoemulsions are composed of oil, surfactant, co-surfactant and water. Especially, cosurfactant plays a critical role in formation of nanoemulsions. In pharmaceutical area, a pre-concentrate form of nanoemulsions which is known as self-nanoemulsifying drug delivery systems (SNEDDS) was available for some water-insoluble drugs. In this study, we investigated the functional behaviors of cosurfactant in design of SNEDDS and nanoemulsions. Cremophor RH $40^{\circledR}$, Propylene carbonate and medium chain triglyceride were selected for surfactant, cosurfactant and oil, respectively. Cyclosporine was employed as a drug. Phase diagrams showed the area of isotropic o/w region which forms o/w nanoemulsions was not significantly affected by the compositional ratio of cosurfactant. But, drug solubilization capacity, droplet size of nanoemulsions and drug release rate were greatly affected by the cosurfactant.
\end{abstract}

Key words - Cyclosporine, Cosurfactant, Nanoemulsions, SNEDDS (self-nanoemulsifying drug delivery system)

Since Hoar and Schulman introduced microemulsions in the 1940s, microemulsions received great interest from scientists because of their unique properties (i.e. small droplet size under $100 \mathrm{~nm}$, low interfacial tension, large surface area, thermodynamic stability and the ability to dissolve insoluble substances, etc (Lawrence et al., 2000). The applications of microemulsions have been still extending further from electrokinetic chromatography to metal oxide nanoparticles synthesis (Bumajdad et al., 2007; Eastoe et al., 2006; Hansen 2003). At the turn of $21^{\text {st }}$ century, paradigm of science rapidly changed from "micro" to "nano". Recently, scientists have referred to these systems as nanoemulsions, which seem more appropriate than microemulsions (Sarker, 2005).

Nanoemulsions show great potentials for drug delivery vehicles which can apply a wide range of drug molecules, cosmetics, diagnostics, and proteins (Porter et al., 2007; Lawrence et al., 2000). However, the practical applications of nanoemulsions were blocked from its large volume (over $10 \mathrm{~mL}$ ). Fortunately, a self-nanoemulsifying drug delivery systems (SNEDDS) which has a reasonable size for softgel encapsulation was

${ }^{\dagger}$ Corresponding Author:

Tel : +82-32-858-9403, E-mail : Sugeun.Yang@Inha.ac.kr

DOI : $10.4333 /$ KPS.2010.40.5.263 developed in the early 1990s (Hauer et al., 1994). SNEDDS spontaneously forms nanoemulsions in the body after a swallowing and enhances the oral bioavailability of drug. SNEDDS is essentially composed of oil, surfactant and cosurfactant with drug. Determination of appropriate components and their compositional ratio is a crucial factor for the formulation of a SNEDDS (Kawakami et al., 2002). Generally, basic components for SNEDDS are selected based on the solubilization capacity for drug. Then, the optimal compositional ratio of oil, surfactant and cosurfactant is determined in phase-diagram studies. The oil of choice is usually a modified or hydrolyzed vegetable oil such as medium chain triglyceride (MCT). MCT has been frequently used because of its high fluidity and compatibility with various surfactants (Constantinides, 1995). MCT also has a higher solubilization capacity for water-insoluble drugs. Non-ionic surfactants with a relatively high hydrophilic and lipophilic balance (HLB) are preferred for surfactant, where polyoxyethylene sorbitan fatty acid esters (i.e., Tween 80) and polyoxyethylene (hydrogenated) vegetable oil (i.e., Cremophor EL, Cremophor RH 40) are the most frequently used.

In most cases, single-chain surfactants alone are unable to reduce the oil/water interfacial tension sufficiently to form nanoemulsions (Lawrence et al., 2000; Attwood, 1994). There- 
fore, cosurfactants such as medium-chain alcohols (i.e., $n$ butanol, $n$-pentanol and $n$-hexanol) are commonly employed (Attwood, 1994).

The functional behaviors of cosurfactants have been reported in many fields. Farago et al. reported that $n$-butanol affected the shape and size of nanoemulsion droplets (Farago et al., 1990). Cosurfactants influenced the crystal formation of $\mathrm{ZnS}$ nanoparticles in nanoemulsions. They synthesized an array of $\mathrm{ZnS}$ nanoparticles with varying morphologies including; spherical, ellipsoidal particles, nanotubes and nanorods by changing aliphatic cosurfactants (T. Charinpanitkul et al., 2005). The area of nanoemulsions was greatly affected by the chemical properties of cosurfactants, where aliphatic alcohols and 1,2-alkanediols with different chain length were selected for cosurfactant (Alany et al., 2000). They proved the area of nanoemulsions gradually decreased as chain length of aliphatic alcohols increased. Interestingly, alkanediols showed the reversed phenomenon that is area of nanoemulsions increased when the longer alkanediols were employed as cosurfactant.

In the pharmaceutical area, only short-chain alcohols such as polyethylene glycol 400, ethanol and propylene glycol are available for the cosurfactant which is generally regarded as safe for human application. Although these cosurfactants have been widely used for the pharmaceutical nanoemulsions, their functional behavior have not been considered. This study examined the pharmaceutical roles of cosurfactant for a design of SNEDDS. Propylene carbonate was selected as a representative cosurfactant. Cremophor RH $40^{\circledR}, \mathrm{MCT}$ and cyclosporine were selected for surfactant, oil and drug, respectively. The effects of propylene carbonate on the characteristics of cyclosporine SNEDDS (i.e., solvent capacity, phase behavior, droplet size and drug release rate) were estimated to determine whether propylene carbonate acts as a solvent for drug or a cosurfactant for formation of nanoemulsions.

\section{Materials and methods}

\section{Materials}

Cyclosporine was kindly supplied form CKD Pharm Co. (Seoul, Korea), polyoxyl hydrogenated castor oil 40 (Cremophor RH $40^{\circledR}$, BASF, Germany), medium chain triglyceride (Sefol ${ }^{\circledR}$, Wellga, Korea), propylene carbonate (Hüls, Germany) were used as supplied. All other chemicals were of reagent grade and used without further purification.

\section{Solubilization capacities of vehicles for cyclosporine}

The solubility of cyclosporine was determined in surfactant alone, cosurfactant alone, oil alone as well as the combination of all these components. Excess cyclosporine was added to 2 $\mathrm{mL}$ of each test mixture, vigorously shaken for 72 hours at $25^{\circ} \mathrm{C}$. Then samples were centrifuged at $15,000 \mathrm{~g}$ for $10 \mathrm{~min}$ to remove the insolubilized drug. An aliquot of supernatant was filtered $(0.45 \mu \mathrm{m})$ and diluted with a 1:1 mixture of methanol and acetone for analysis. The concentration of cyclosporine was determined by HPLC (Alliance 2610, Waters, MA) using a Capcellpak $\mathrm{C}_{8}$ column (UG120, Shiseido, Japan) at $70^{\circ} \mathrm{C}$, the mobile phase consisted of $65 \% \mathrm{v} / \mathrm{v}$ acetonitrile, $15 \%$ $\mathrm{v} / \mathrm{v}$ methanol, $20 \% \mathrm{v} / \mathrm{v}$ water, and $1 \%$ phosphoric acid at a flow rate of $1 \mathrm{~mL} / \mathrm{min}$. The effluent was monitored at $210 \mathrm{~nm}$.

\section{Preparation of pseudo-ternary phase diagrams}

Pseudo-ternary phase diagrams were constructed to identify self nano-emulsifying regions and to select suitable concentrations of oil, surfactant and cosurfactant for the formulation of SNEDDS. At first, different mixtures of surfactant and cosurfactant $(\mathrm{Su} / \mathrm{Cos})$ in the various weight ratios (i.e., 2:1, 3:1, 4:1) were prepared. Each surfactant-cosurfactant mixtures ( $\mathrm{Su} /$ $\mathrm{Cos}$ ) was then vortex-mixed with oil (MCT) to yield varying ratios of $\mathrm{Su} / \mathrm{Cos}$ and MCT (i.e., 9:1, 8:2, 7:3, 6:4, 5:5, 4:6, 3:7, $2: 8,1: 9$ by weight). Finally, the resulting mixtures were titrated with water, vortex-mixed, equilibrated overnight at room temperature. The clear, transparent nanoemulsion area was visually determined and named isotropic regions $\left(\mathrm{I}_{\mathrm{w}}\right.$; isotropic water region and $\mathrm{I}_{0}$; isotropic oil region).

\section{Droplet size analysis}

The compositional effect of oil, surfactant and cosurfactant on the droplet size of nanoemulsions was studied. The various mixtures of oil, surfactant and cosurfactant were made for the test. For example, to see the effect of cosurfactant on droplet size, propylene carbonate was gradually added at the same mixture of surfactant and oil. The final mixtures with different content of cosurfactant were introduced to distilled water $\left(50 \mathrm{~mL}, 37^{\circ} \mathrm{C}\right)$ in beakers. The water was then gently shaken by magnetic stirring for $30 \mathrm{~min}$ to form nanoemulsions. Droplet size was determined by dynamic light scattering (DLS) instrument (NICOMP 370, Agilent Technologies, CA) equipped with a $15 \mathrm{~mW}$ laser diode and PMT detector with an optical fiber set at 90 degrees.

\section{Drug release study}

The effect of cosurfactant on the drug release was estimated following the reported method for SNEDDS (Kim et al., 2001). The test formulas containing the different amount of cosurfactant were filled into empty hard gelatin capsules (\#000) and dropped into $900 \mathrm{~mL}$ of simulated gastric juice 
without pepsin (pH 1.2) at $37 \pm 0.5^{\circ} \mathrm{C}$, under paddle stirring of $50 \mathrm{rpm}$. At fixed time interval, aliquots of dissolution medium $(5 \mathrm{~mL})$ were withdrawn, and immediately filtered $(0.45 \mu \mathrm{m})$ for analysis. The amount of cyclosporine in the sample was assayed by using HPLC.

\section{Result and Discussion}

\section{Effect of cosurfactant on solubilization capacity of SNEDDS}

The solubility of cyclosporine was $13.2 \pm 2.4 \mathrm{mg} / \mathrm{g}$ in MCT, $10.6 \pm 1.6 \mathrm{mg} / \mathrm{g}$ in Cremophor RH $40^{\circledR}$ and $285.3 \pm 8.5 \mathrm{mg} / \mathrm{g}$ in propylene carbonate, respectively. Propylene carbonate showed much higher solubilization capacity against cyclosporine than other components. The solubility of cyclosporine in oil-surfactant mixture was not changed as the concentration of surfactant increased in the range of $20 \sim 60 \%$ and then slightly decreased thereafter (Figure 1A). In contrast, the solubility of cyclosporine in oil-cosurfactant mixture linearly increased as the content of propylene carbonate increased (Figure 1B). Figure $1 \mathrm{C}$ also shows that the solubility of cyclosporine in the oil/ surfactants/cosurfactant mixtures linearly decreased as the relative ratio of cosurfactant to surfactant decreased. In general, the solubility of cyclosporine is highly dependent on the presence of propylene carbonate. For the conventional dosage design, at least $100 \mathrm{mg}$ of cyclosporine should be dissolved in $1.0 \mathrm{~g}$ of SNEDDS. In this respect, propylene carbonate proved to have a solvent capacity sufficient to dissolve $100 \mathrm{mg}$ of cyclosporine in $1 \mathrm{~g}$ of SNEDDS.

\section{Effect of cosurfactant on phase behavior}

Once the appropriate surfactant, cosurfactant and oil have been selected, phase diagrams are usually prepared to screen formulations. A typical phase diagram is constructed with one axis representing water, a second axis representing oil (MCT),

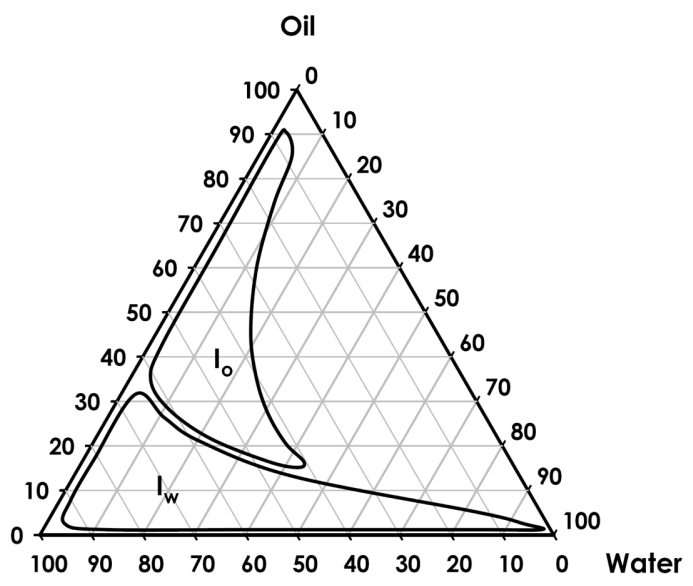

Figure 2. Typical pseudo-ternary phase diagram constructed with oil, mixture of surfactant and cosurfactant $(\mathrm{Su} / \mathrm{Cos}, 2: 1)$, and water, $\mathrm{I}_{\mathrm{w}}$; isotropic water region, $\mathrm{I}_{\mathrm{o}}$; isotropic oil region.

and the third representing surfactant-cosurfactant mixture (2:1, $\mathrm{w} / \mathrm{w}$ ), as shown in Figure 2. Approximately, $45 \%$ of the area on the diagram was found to be the isotropic region. The wider the isotropic region means the more stable the formation of nanoemulsions and the more stable absorption of drug in the gastrointestinal tract, which is normally more variable from food intake and digestive juice secretion. The relative area of isotropic region on phase diagrams was not affected by varying the ratio of surfactant to cosurfactant (1.5:1 to 3:1) (Figure 3). Our phase diagram studies did not show that propylene carbonate functioned as cosurfactant, affecting the area of isotropic region.

Effect of cosurfactant on droplet size of nanoemulsions

The droplet size is one of the most important factors in emulsion chemistry, as it affects the stability of emulsions (Charman et al., 1992) and the absorption of drug (Tarr et al., 1989). As shown in Figure 4A, droplet size significantly
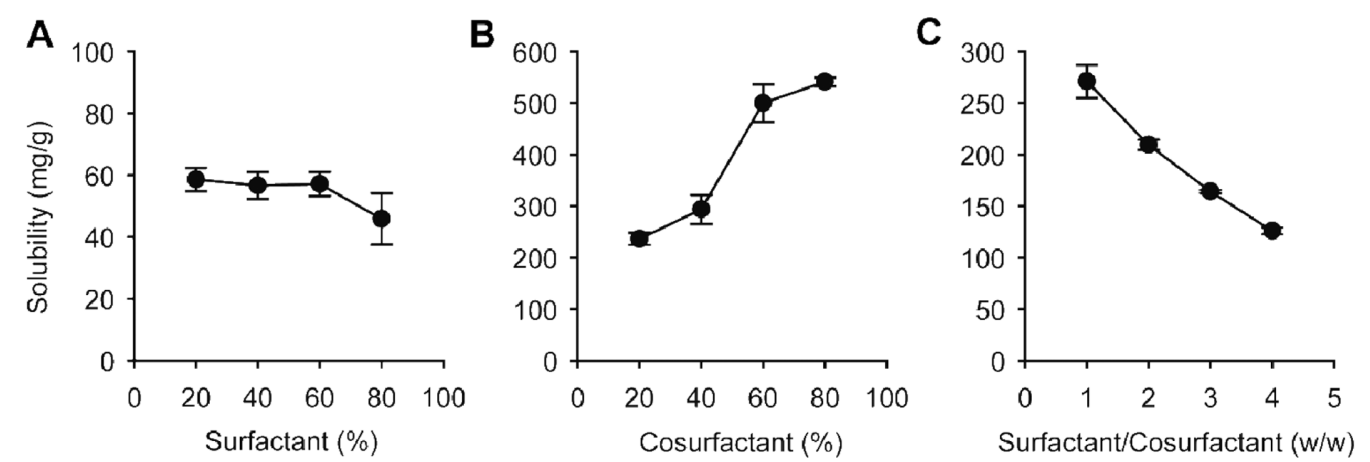

Figure 1. Effect of surfactant (A), cosurfactant (B) and compositional ratio of surfactant and cosurfactant (C) on the solubility of cyclosporine in the mixture of oil and surfactant (A), in the mixture of oil and cosurfactant (B) and in the mixture of oil, surfactant and cosurfactant (C). 


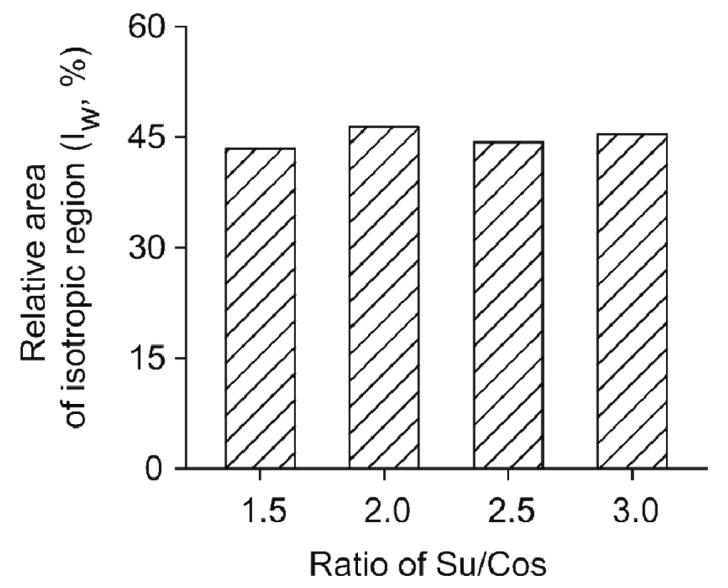

Figure 3. Relative areas of isotropic region in the phase diagram, constructed with the different ratios of surfactant/cosurfactant $(\mathrm{Su}$ Cos).

increased when the content of propylene carbonate increased over 35\% (w/w) of SNEDDS. Figure 4B shows the effect of oil on the droplet size of nanoemulsions at different ratios of surfactant and cosurfactant. As oil content is increased in the nanoemulsion system, droplet size increased linearly. The 2.5:1 ratio of surfactant to cosurfactant formed the most stable nanoemulsion with steady droplet size regardless of the content of oil when compared to the other ratios tested. Cyclosporine in nanoemulsions did not affect the droplet size up to 150 $\mathrm{mg} / \mathrm{g}$ of concentration (Figure $4 \mathrm{C}$ ). In general, propylene carbonate had the greatest affect on droplet size of nanoemulsion. These data suggest that adjusting the most suitable content of cosurfactant is one of the critical factors in the design of SNEDDS.

\section{Effect of cosurfactant on drug release}

The release of cyclosporine from SNEDDS was rapid since more than $80 \%$ of cyclosporine consistently dissolved within

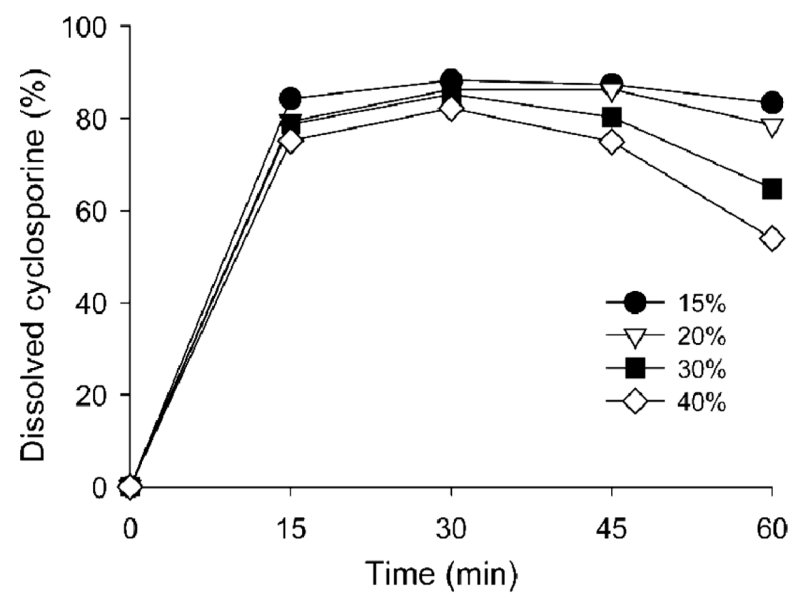

Figure 5. Effect of the content of cosurfactant $(\%, w / w)$ in SNEDDS on the release of cyclosporine.

30 minutes in all test formulas (Figure 5). However, dissolution studies showed that the release rate decreased as the concentration of cosurfactant increased. The release rate of test formulas with higher concentrations of cosurfactant (i.e., $40 \%$ content of propylene carbonate) began to drop after 30 minutes of dissolution. These results are consistent with droplet size studies that show the mean droplet size increased by increasing the concentration of cosurfactant (Figure 4A). However, the decrease in dissolution rate after 30 minutes cannot be fully explained by the droplets size. Hydrophilic cosurfactants such as propylene carbonate, which highly contributes to the solubilization capacity of SNEDDS for hydrophobic drug, substantially separates from oil-droplets after formation of nanoemulsions in water, and drug precipitates out due to defection of solvent capacity. Similar apprehensions were mentioned in some literatures that drug might precipitate out from nano-droplets by the same mechanism (Gershanik et al., 2000; Lawrence et al., 2000).

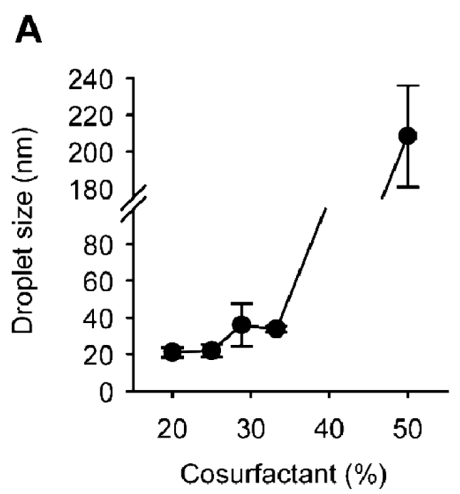

B

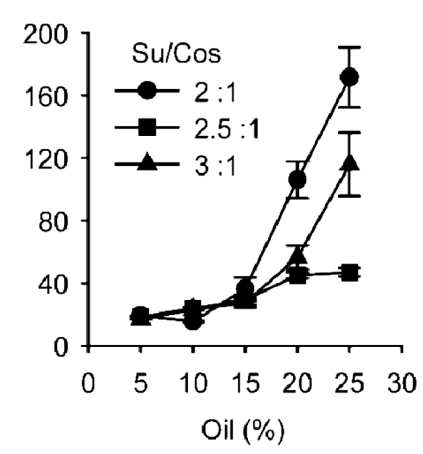

\section{C}

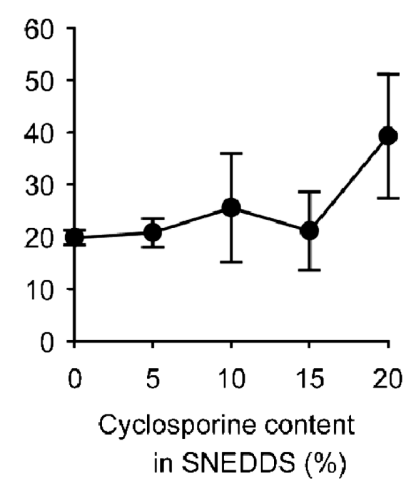

Figure 4. Effect of cosurfactant (A) and oil (B) and cyclosporine (C) on the mean droplet size of nanoemulsions, 2:1, 2.5:1 and 3:1 in panel (B) represent the mixing ratio of surfactant and cosurfactant. 


\section{Optimized composition for cyclosporine SNEDDS}

Increasing the ratio of surfactant to cosurfactant results in a reduced solvent capacity for drug and elevates the volume needed which may be disadvantageous for swallowing. The amount of surfactant should be decreased when SNEDDS spontaneously form nanoemulsions. Our cosurfactant solubility studies indicate that the higher compositional ratio of cosurfactant results in an increase in the solubility of drugs in SNEDDS. However, when considering droplet size and dissolution rate, the content of cosurfactant should be decreased to produce a more stable nanoemulsions. Generally, the optimal formula for SNEDDS can be acquired from phase-diagram studies. Figure 2 demonstrates that 10 to $20 \%$ of oil composition with the 2:1 mixture of surfactant and cosurfactant is ideal for forming stable $\mathrm{o} / \mathrm{w}$ nanoemulsions $\left(\mathrm{I}_{\mathrm{w}}\right.$ phase).

\section{Conclusion}

As previously mentioned, nanoemulsions have been extensively studied in various areas of industrial and academic fields because of their various applications. Most of the studies have employed either hydrocarbon oils or cyclic oils (i.e., heptane, dodecane or cyclohexane) as oil, and selected hydrophobes of 12 carbons (i.e, sodium dodecyl sulphate (SDS), sodium lauryl sulfate (SLS) and tetraethylene glycol monododecyl ether $\left(\mathrm{C}_{12} \mathrm{E}_{4}\right)$ ) as surfactant (Lawrence et al., 2000). In these systems, cosurfactant such as butanol and isopropanol are required to reduce the interfacial tension and to enhance the miscibility between oil (i.e., hydrocarbon oils) and water (Alany et al., 2000; Lawrence et al., 2000).

In our studies we demonstrated that cosurfactants should be reconsidered for their ability to dissolve drugs in emulsion systems for pharmaceutical applications. Propylene carbonate which was selected as a cosurfactant for cyclosporine SNEDDS highly enhanced the solvent capacity of SNEDDS, and critically affected the droplet size of nanoemulsions and the dissolution rate of cyclosporine.

\section{Acknowledgements}

This work was supported by the Korea Research Foundation Grant funded by the Korean Government (MOEHRD). (KRF2006-214-E00039)

\section{References}

Alany, R.G., Rades, T., Agatonovic-Kustrin, S., Davies, N.M., Tucker, I.G., 2000. Effects of alcohols and diols on the phase behaviour of quaternary systems. Int. J. Pharm. 196, 141145.

Attwood, D., 1994. Microemulsions. In: Colloidal Drug Delivery Systems. Marcel Dekker, New York.

Bumajdad, A., Eastoe, J., Zaki, M.I., Heenan, R.K., Pasupulety, L., 2007. Generation of metal oxide nanoparticles in optimised microemulsions. J. Colloid Interface Sci. 312, 68-75.

Charman, S.A., Charman, W.N., Rogge, M.C., Wilson, T.D., Dutko, F.J., Pouton, C.W., 1992. Self-emulsifying drug delivery systems: formulation and biopharmaceutic evaluation of an investigational lipophilic compound. Pharm. Res. 9, 87-93.

Constantinides, P. P., 1995. Lipid microemulsions for improving drug dissolution and oral absorption: physical and biopharmaceutical aspects. Pharm. Res. 12, 1561-1572.

Eastoe, J., Hollamby, M.J., Hudson, L., 2006. Recent advances in nanoparticle synthesis with reversed micelles. Adv. Colloid Interface Sci. 128-130, 5-15.

Farago, B., Richter, D., Huang, J.S., Safran, S.A., Milner, S.T., 1990. Shape and size fluctuations of microemulsion droplets: The role of cosurfactant. Phys. Rev. Lett. 65, 3348-3351.

Gershanik, T., Benita, S., 2000. Self-dispersing lipid formulations for improving oral absorption of lipophilic drugs. Eur. J. Pharm. Biopharm. 50, 179-188.

Hansen, S.H., 2003. Recent applications of microemulsion electrokinetic chromatography. Electrophoresis 24, 3900-3907.

Hauer, B., Meinzer, A., Posanski, U., Richter, F., 1994. Pharmaceutical compositions comprising cyclosporins. United States Patent 5342625.

Kawakami, K., Yoshikawa, T., Moroto, Y., Kanaoka, E., Takahashi, K., Nishihara, Y., Masuda, K., 2002. Microemulsion formulation for enhanced absorption of poorly soluble drugs. I. Prescription design. J. Control. Release 81, 65-74.

Kim, C.K., Cho, Y.J., Gao, Z.G., 2001. Preparation and evaluation of biphenyl dimethyl dicarboxylate microemulsions for oral delivery. J. Control. Release 70, 149-155.

Lawrence, M.J., Rees, G.D., 2000. Microemulsion-based media as novel drug delivery systems. Adv. Drug Deliv. Rev. 45, 89121.

Porter, C.J., Trevaskis, N.L., Charman, W.N., 2007. Lipids and lipid-based formulations: optimizing the oral delivery of lipophilic drugs. Nat. Rev. Drug Discov. 6, 231-248.

Sarker, D.K., 2005. Engineering of nanoemulsions for drug delivery. Curr. Drug Deliv. 2, 297-310.

Charinpanitkul, T., Chanagul, A., Dutta, J., Rungsardthong, U., Tanthapanichakoon, W., 2005. Effects of cosurfactant on ZnS nanoparticle synthesis in microemulsion. Sci. Tech. Adv. Mat. 6, 266-271.

Tarr, B.D., Yalkowsky, S.H., 1989. Enhanced intestinal absorption of cyclosporine in rats through the reduction of emulsion droplet size. Pharm. Res. 6, 40-43. 